\title{
Incorporating anisotropy in the Barcelona basic model
}

\author{
Muayad A. Al-Sharrad ${ }^{1, a}$ and Domenico Gallipoli ${ }^{2}$ \\ ${ }^{1}$ Department of Civil Engineering, University of Anbar, Ramadi 31001, Iraq \\ ${ }^{2}$ Université de Pau et des Pays de l'Adour, Anglet, France
}

\begin{abstract}
The paper presents an elasto-plastic model for unsaturated soils that extends the Barcelona basic model to introduce a dependency of mechanical behaviour on material anisotropy. The model builds upon a previous proposal by Al-Sharrad and Gallipoli [1] by linking material anisotropy to both the distortion and aspect ratio of the elliptical yield curve in the constant suction plane of deviator versus mean net stress. In order to take into account anisotropic behaviour, an additional constitutive equation is therefore proposed to uniquely relate the aspect ratio and the distortion of the yield ellipse. The new model is calibrated and validated against the experimental data by Al-Sharrad [2] via the simulation of triaxial tests conducted at various suctions on compacted kaolin samples. These simulations show that the new model predicts yield stresses and shear strains during anisotropic loading with greater accuracy compared to the model of Al-Sharrad and Gallipoli [1].
\end{abstract}

\section{Introduction}

Anisotropic soils exhibit properties that depend on the direction along which these properties are measured. The anisotropic mechanical behaviour of saturated soils has been studied since the 1970s (e.g. [3, 4, 5, 6]) and several elasto-plastic models, which incorporate directional dependency of stress-strain behaviour, have since then been proposed (e.g. [7, 8]). Similar studies have also been undertaken for unsaturated soils (e.g. [9, 10, 11]), though relatively fewer models have been proposed in this case (e.g. $[12,13,14,2,1])$. A review of existing elastoplastic anisotropic models for both saturated and unsaturated soils can be found in Al-Sharrad [2].

In the present work, the Barcelona basic model (BBM) for unsaturated soils is modified to incorporate a dependency of yielding behaviour on material anisotropy. The new model is referred as ABBM3 because it builds upon previous proposals by Al-Sharrad and Gallipoli [1] and D'Onza et al. [13] which are referred as ABBM2 and ABBM1, respectively. The formulation of ABBM3 is similar to that of ABBM2 with one important difference consisting in the definition of the elliptical yield curve in the constant suction plane of deviator stress versus mean net stress. Unlike ABBM2, ABBM3 links material anisotropy not only to the distortion but also to the aspect ratio of the constant suction yield ellipse via an additional constitutive relationship between aspect ratio and distortion. By assuming an associated flow rule, ABBM3

\footnotetext{
$\overline{{ }^{a} \text { Corresponding author: an_mission } @ y a h o o . c o m ~}$
}

provides improved predictions of shear strains and yield stresses compared to ABBM2.

\section{Overview of ABBM2}

The ABBM2 is formulated in terms of mean net stress $p$, deviator stress $q$ and suction $s$. The model is capable of simulating the elasto-plastic anisotropic behaviour of unsaturated soils and reduces to BBM in the case of material isotropy. In particular, ABBM2 assumes that fabric anisotropy develops as a consequence of both plastic volumetric and plastic shear strains.

The yield curve takes the form of a distorted ellipse in the constant suction $q: p$ plane (see Figure 1):

$$
f=\left(q-\alpha\left(p+p_{s}(s)\right)\right)^{2}-\left(M^{2}-\alpha^{2}\right)\left(p+p_{s}(s)\right)\left(p_{m}(s)-p\right)=0
$$

where $M$ is the slope of the critical state while $p_{m}(s)$ and $\alpha$ are the size and distortion of the yield ellipse, respectively (note that $\alpha$, unlike $p_{m}(s)$, is independent of suction). Similar to BBM, ABBM2 assumes a critical state line in the constant suction $q: p$ plane defined as:

$$
q= \pm M\left[p+p_{s}(s)\right]
$$

where the slope $M$ of the critical state line controls also the aspect ratio of the distorted yield ellipse, which is equal to $\left(M^{2}-\alpha^{2}\right)^{1 / 2}$. Likewise $\mathrm{BBM}, p_{s}(s)$ defines the common intercept of both the yield ellipse and the critical state line with the negative $p$ axis. This intercept varies 
non-linearly with suction as proposed by Stropeit et al. [12]:

$$
p_{s}(s)=a\left(1-\exp \frac{-s}{a}\right)
$$

where $a$ is a model parameter.

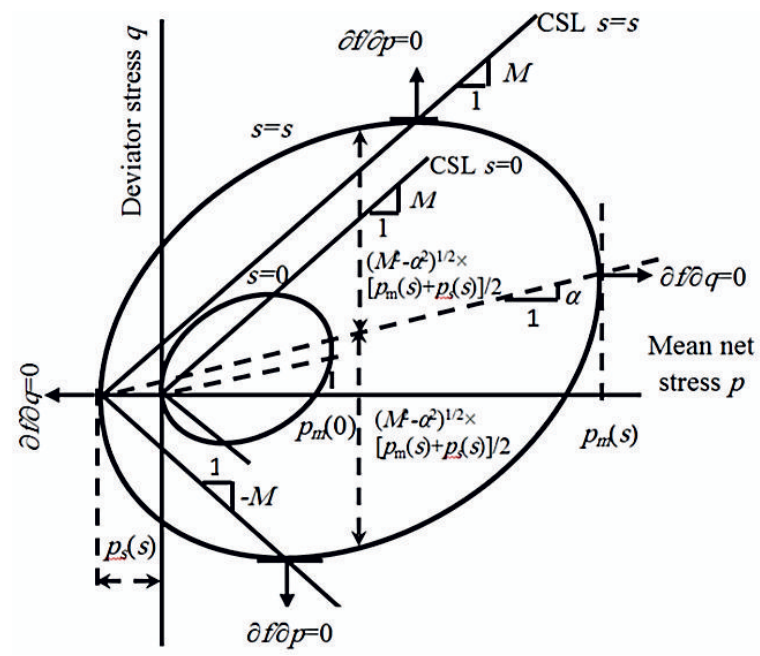

Figure 1. ABBM2 yield ellipses in constant suction $q: p$ plane.

The model implements an associated plastic flow rule which, together with Equation 1, results in the following relationship between plastic volumetric strain $\varepsilon_{v}^{p}$ and plastic shear strain $\varepsilon_{s}^{p}$ :

$$
\frac{d \varepsilon_{s}^{p}}{d \varepsilon_{v}^{p}}=\frac{2(\eta-\alpha)}{\left(M^{2}-\eta^{2}\right)}
$$

where $\eta$ is the stress ratio defined as:

$$
\eta=\frac{q}{p+p_{s}(s)}
$$

Equation (4) predicts, at the intersection between the yield ellipse and the critical state line, a plastic flow vector that is always vertical (i.e. zero plastic volumetric strains), regardless of whether the soil is actually at critical state. This condition is however unnecessary because the flow vector only needs to be vertical if the soil is actually at critical state, and will therefore be relaxed in $\mathrm{ABBM}$.

In $\mathrm{ABBM} 2$, the volumetric hardening law is identical to that of BBM and relates the size of the yield ellipse $p_{m}(s)$ to plastic volumetric strains $\varepsilon_{v}^{p}$. A kinematic hardening law is also introduced to define the evolution of anisotropy by linking the changing distortion $\alpha$ of the yield ellipse to the increments of plastic volumetric and shear strains $\varepsilon_{v}^{p}$ and $\varepsilon_{s}^{p}$ :

$$
d \alpha=\mu[f(\eta)-\alpha]\left[\left(d \varepsilon_{v}^{p}\right)^{2}+\frac{9}{2}\left(d \varepsilon_{s}^{p}\right)^{2}\right]^{1 / 2}
$$

where $\mu$ is a model parameter and $f(\eta)$ is a tri-linear relationship defined as:

$$
\begin{aligned}
& f(\eta)=-c M \text { for } \eta \leq-M \\
& f(\eta)=c \eta \text { for }-M<\eta<M \\
& f(\eta)=c M \text { for } \eta \geq M
\end{aligned}
$$

where $c$ is a model parameter. According to Equations 5 and 6 , if the soil is loaded with a stress ratio equal or greater than the critical one, i.e. $|\eta| \geq M$, the distortion of the yield ellipse will tend towards the following critical state value:

$$
\left|\alpha_{c s}\right|=c M
$$

Note that stress ratio $\eta$ and distortion $\alpha$ are positive for triaxial compression and negative for triaxial extension.

\section{Additional assumptions in ABBM3}

Al-Sharrad el al. [11] showed that, for a given degree of anisotropy, experimental yield points are best fitted in a constant suction $q: p$ plane by a distorted ellipse with an aspect ratio $m$ generally greater than $\left(M^{2}-\alpha^{2}\right)^{1 / 2}$, which is the aspect ratio predicted by ABBM2. A further improvement is obtained if different aspect ratios are used for the upper (compression) section and the lower (extension) section of the ellipse (see Figure 2), i.e. if $m=$ $m_{c}$ for the section above the vertical tangent point $(\eta>\alpha)$ and $m=m_{e}$ for the section below the vertical tangent point $(\eta<\alpha)$. Therefore, unlike ABBM2, ABBM3 assumes constant suction yield ellipses with a different aspect ratio $m$ :

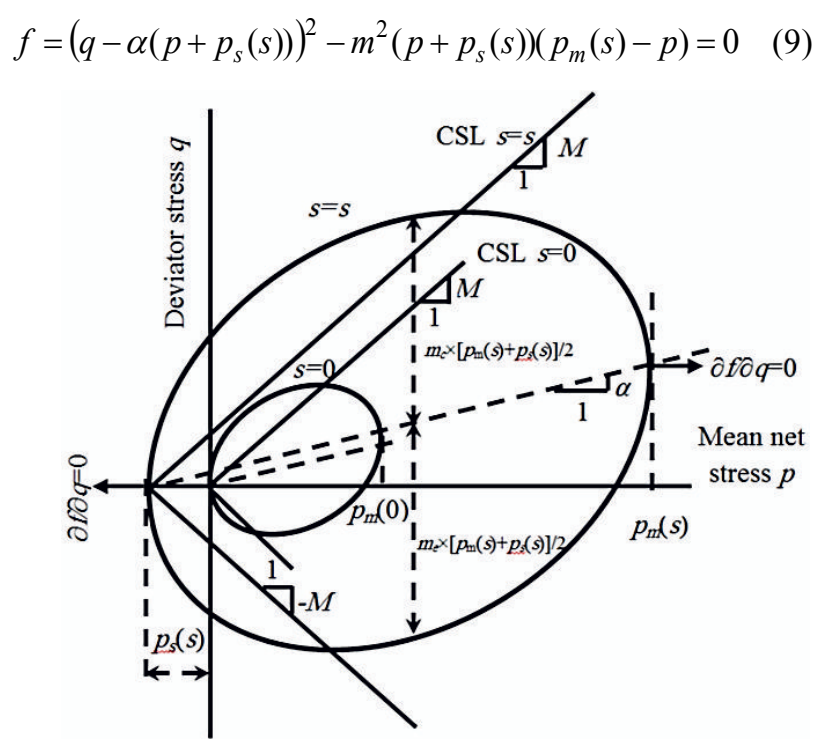

Figure 2. ABBM3 yield ellipses in constant suction $q: p$ plane.

By assuming an associated plastic flow rule together with Equation 9, the following relationship between plastic strains is obtained in ABBM3:

$$
\frac{d \varepsilon_{s}^{p}}{d \varepsilon_{v}^{p}}=\frac{2(\eta-\alpha)}{\left(m^{2}+\alpha^{2}-\eta^{2}\right)}
$$


If critical state conditions are imposed in Equation 10, i.e. if $\frac{d \varepsilon_{s}^{p}}{d \varepsilon_{v}^{p}}=0,|\eta|=M$ and $|\alpha|=\left|\alpha_{c s}\right|=c M$, the following aspect ratio at critical state is obtained:

$$
m_{c s}=\left(M^{2}-\alpha_{c s}^{2}\right)^{1 / 2}=M\left(1-c^{2}\right)^{1 / 2}
$$

The aspect ratio must be equal to $m_{c s}$ when the soil attains critical state in order to ensure a vertical direction for the plastic flow vector as required by the definition of critical state. Due to the assumption of a different aspect ratio than in $\mathrm{ABBM} 2$, in $\mathrm{ABBM} 3$ the plastic flow vector is generally not vertical at the intersection between the yield ellipse and critical state line but becomes vertical only when the soil attains critical state.

\section{Model performance}

Most model parameters in ABBM3 are the same as in ABBM2, namely $\kappa, \kappa_{s}, G, \lambda(0), \beta, r, N(0), p^{c}, a, M, \mu$ and $c$. In particular, $\kappa$ is the elastic swelling coefficient associated to stress, $\kappa_{s}$ the elastic swelling coefficient associated to suction, $G$ is the elastic shear modulus, $\lambda(0)$ is the slope of the saturated normal compression line, $\beta$ is a parameter defining the change of the slope $\lambda(s)$ of constant suction normal compression lines with suction, $r$ is a parameter defining the limit value of $\lambda(s)$ when suction tends to infinity and $N(0)$ is the specific volume at a reference pressure $p^{c}$ on the saturated normal compression line. Parameters $a, M, \mu$ and $c$ have been previously defined in Equations 2, 3, 6 and 7.

The values of parameters $\kappa, \kappa_{s}, G, \lambda(0), r, \beta, N(0), p^{c}$, $a$ and $M$ are independent of the particular form of the yield ellipse. Therefore, the same values presented in [1] for $\mathrm{ABBM} 2$ were also assumed for $\mathrm{ABBM} 3$. The values of parameters $\mu$ and $c$ were instead specifically obtained in this work by following the iterative calibration procedure of [2]. This procedure involves performing model simulations with trial values of $\mu$ and $c$ along predefined stress paths. The computed and experimental changes of $\alpha$, caused by plastic straining along this stress path, are then compared and, in case of discrepancy, the values $\mu$ and $c$ are refined until a satisfactory match is obtained. All the above parameter values are listed in Table 1.

For isotropically compacted samples, the initial value of the volumetric hardening parameter $p_{m}(0)$ was calculated as $22 \mathrm{kPa}$ by following the procedure outlined in [15]. For anisotropically compacted samples, the initial value of the volumetric hardening parameter $p_{m}(0)$ was also calculated as $22 \mathrm{kPa}$ according to the work of AlSharrad [2] by fitting the yield ellipse of Equation 9 to the yield data point at $s=0$. Similarly, the initial distortion $\alpha$ of the yield ellipse was calculated as 0 and 0.21 for isotropically and anisotropically compacted samples, respectively, based the work of Al-Sharrad [2].

These initial values of $p_{m}(0)$ and $\alpha$, together with the parameter values in Table 1 , were employed for simulating the behaviour of both isotropically and anisotropically compacted specimens during triaxial tests. However, due to space limitations, only few simulations of the behaviour of the isotropically compacted samples are shown later in this paper.

\subsection{Additional parameters in ABBM3}

In ABBM3 the aspect ratio of the yield ellipse is related to the current degree of anisotropy by means of an additional constitutive relationship between the aspect ratio and the distortion of the yield ellipse. In order to define this relationship, the experimental values of aspect ratios in compression and extension, i.e. $m_{c}$ and $m_{e}$, are plotted in Figure 3 against the corresponding values of distortion $\alpha$.

Equations 8 and 11, together with the parameter values in Table 1, calculate the critical state values of distortion and aspect ratio as $\left|\alpha_{c s}\right|=0.30$ and $m_{c s}=0.67$. Therefore, Figure 3 covers the entire range of distortion values from isotropic $(\alpha=0)$ up to critical state. Inspection of Figure 3 also indicates that the experimental values of $m_{c}$ and $m_{e}$ are practically constant over the measured range and equal to the isotropic aspect ratios $m_{0 c}=0.85$ and $m_{0 e}=1.17$, respectively.

The additional constitutive law must therefore predict an approximately constant value of aspect ratio over a relatively large range of distortions, followed by a sharp decrease towards $m_{c s}$ when the distortion tends to $\left|\alpha_{c s}\right|$. The function chosen to provide this form of behaviour is:

$$
m^{2}=\left[m_{0}^{2}-M^{2}\left(1-c^{2}\right)\right] \tanh \frac{F(c M-|\alpha|)}{|\alpha|}+M^{2}\left(1-c^{2}\right)
$$

where $m_{0}$ is the isotropic aspect ratio at $\alpha=0$ (either $m_{0 c}$ or $m_{0 e}$ ) and $F$ is an additional model parameter controlling the rate of variation of aspect ratio. The predictions by Equation 12 are plotted in Figure 3 for three different values of $F$. A value of $F=20$, or even greater, is required to fit the experimental data. However, preliminary simulations (not shown in the paper) indicated that large values of $F$ result in the over-prediction of peak deviator stress during shearing. On the contrary, values of $F \leq 5$ provide better predictions of shear strains especially if triaxial extension tests are simulated. Consequently, a value of $F=5$ was selected in the current work.

In summary, ABBM3 requires three extra parameters, namely $m_{0 c}, m_{0 e}$ and $F$, in addition to the twelve of ABBM2 (Table 1).

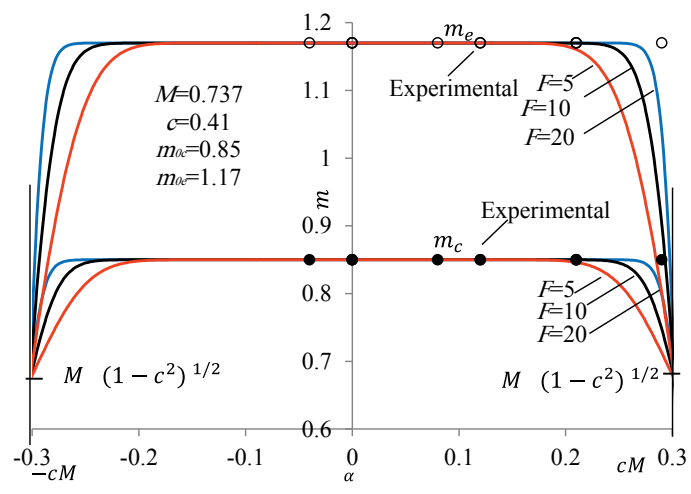

Figure 3. Variation of aspect ratio $m$ with distortion $\alpha$. 
Table 1. Parameter values.

\begin{tabular}{|c|c|c|c|}
\hline$\kappa$ & 0.012 & $M$ & 0.737 \\
\hline$\kappa_{s}$ & 0.004 & $a$ & $216.6 \mathrm{kPa}$ \\
\hline$G$ & $9960 \mathrm{MPa}$ & $\mu$ & 6.6 \\
\hline$\lambda(0)$ & 0.157 & $c$ & 0.41 \\
\hline$N(0)$ & 1.80 & $m_{0 c}$ & 0.85 \\
\hline$r$ & 4.55 & $m_{0 e}$ & 1.17 \\
\hline$\beta$ & $0.0008 \mathrm{kPa}^{-1}$ & $F$ & 5 \\
\hline$p^{c}$ & $696 \mathrm{kPa}$ & & \\
\hline
\end{tabular}

\subsection{Predicted yield surface}

Figure 4 shows the experimental yield stresses measured by Al-Sharrad [2] together with the yield ellipses predicted by ABBM3 and ABBM2 using the parameter values of Table 1. In each of the three plots in Figure 4, every yield point is obtained from a different test on ostensibly the same material. Specimens were initially equalized at $s=300 \mathrm{kPa}$ and then subjected to a load/unload cycle at a constant stress ratio of $\Delta q / \Delta p=1.2$ (Figure $4 \mathrm{a}$ ), $\Delta q / \Delta p=0$ (Figure $4 \mathrm{~b}$ ) and $\Delta q / \Delta p=-1$ (Figure $4 \mathrm{c})$. The load/unload cycle ranged from a small initial stress to $p=200 \mathrm{kPa}$, which produces significant plastic deformations and hence a noticeable modification of material anisotropy (as confirmed by the differences between Figures 4a, 4b and 4c). Afterwards, specimens were sheared along different stress ratios to measure distinct points on the yield surface.

Inspection of Figure 4 indicates that ABBM3 provides, as expected, a better match to the experimental point than $\mathrm{ABBM} 2$. In particular, $\mathrm{ABBM} 3$ accurately predicts both the aspect ratio and the distortion of the yield ellipse, though it slightly under-predicts its size in Figures $4 \mathrm{~b}$ and $4 \mathrm{c}$. In contrast, ABBM2 significantly over-predicts the size of the yield ellipse in Figures $4 \mathrm{a}$ and $4 \mathrm{c}$. Also, the aspect ratio predicted by ABBM2 is smaller compared to the experimental one.

\subsection{Stress-strain behaviour}

The performance of ABBM3 was investigated by simulating a number of triaxial tests conducted by AlSharrad [2] on specimens of isotropically compacted kaolin.

Figures 5 and 6 show the same set of tests in the $v: \ln p$ and $q: \varepsilon_{s}$ planes, respectively, where $v$ is the specific volume and $\varepsilon_{s}$ is the true shear strain. Three specimens were equalized at $s=300 \mathrm{kPa}, 100 \mathrm{kPa}$ and 0 (saturated), respectively, and then isotropically loaded at constant suction to $p=300 \mathrm{kPa}$ before being sheared to failure at constant confining pressure. Figures $5 \mathrm{a}$ and $6 \mathrm{a}$ compare the predictions of ABBM3 and BBM while Figures 5b and $6 \mathrm{~b}$ compare the predictions of ABBM3 and ABBM2. The predictions during isotropic loading by the three models are undistinguishable and agree well with the experimental data. During shearing, the experiments show a post-peak reduction of deviator stress caused by strain localization (observed in all tests). This experimental response is better captured by ABBM3 than BBM and ABBM2 (Figure 6). The better performance of ABBM3 is due to the assumed evolution of the yield ellipse with changing anisotropy in combination with an associated flow rule, which leads to the prediction of smaller shear strains compared to BBM and ABBM2. In many instances, ABBM3, unlike the other two models, is capable of predicting the observed peak of deviator stress during shearing as well as the subsequent reduction of deviator stress towards critical state. This happens when the stress point crosses the critical state line while the distortion and aspect ratio of the yield curve have not yet attained their respective critical state values calculated by Equations 8 and 11. Because of this, positive (i.e. compressive) plastic volumetric strains are still being predicted and the yield curve therefore keeps expanding which allows the stress state to cross the critical state line. Finally, as shearing progresses, the stress path converges back on the critical state line. At critical state, all models predict the same value of deviator stress $q$ which is, in most cases, close to the experimental value.

Figures 7 and 8 show another set of tests in the $v: \ln p$ and $q: \varepsilon_{s}$ planes, respectively. In this case, three specimens were equalized at $s=300 \mathrm{kPa}, 100 \mathrm{kPa}$ and 0 (saturated) and then sheared to failure with a stress ratio $\Delta q / \Delta p=1$ under constant suction. As before, Figures $7 \mathrm{a}$ and $8 \mathrm{a}$ compare the predictions of ABBM3 and BBM while Figures $7 \mathrm{~b}$ and $8 \mathrm{~b}$ compare the predictions of $\mathrm{ABBM} 3$ and ABBM2. Simulations in the $v: \ln p$ plane (Figure 7) indicate that all models predict relatively well the yielding points marked by the sharp change in the slope of the compression curves. The slope of the post-yield compression line and the critical state values of specific volume are also reasonably captured by ABBM2 and ABBM3 but not BBM, which grossly over-predicts both of them. 

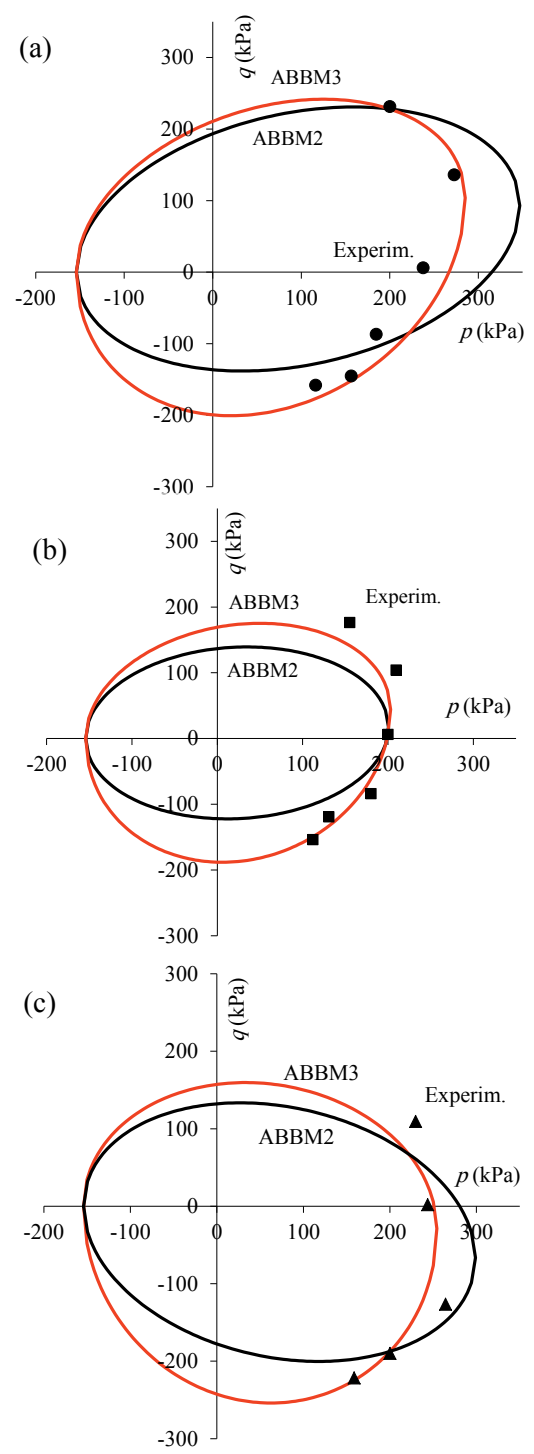

\section{Conclusions}

An elasto-plastic anisotropic model for unsaturated soils, named ABBM3, has been presented. ABBM3 modifies the well-known BBM to incorporate material anisotropy and enhances a previous anisotropic model, named

ABBM2, proposed by the same authors [1].

Figure 4. Experimental yield points and yield surfaces predicted by ABBM3 and ABBM2 for three soils with variable degrees of induced anisotropy.
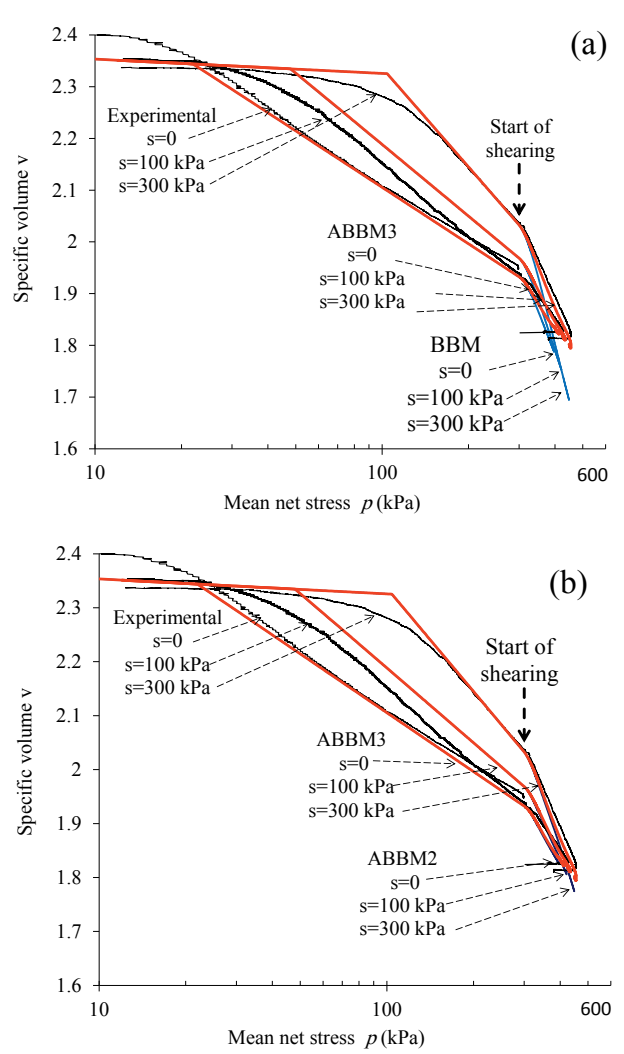

Figure 5. Experimental and predicted variation of $v$ against $\ln p$ : (a) $\mathrm{ABBM} 3$ vs $\mathrm{BBM}$ and (b) $\mathrm{ABBM} 3$ and $\mathrm{ABBM} 2$.

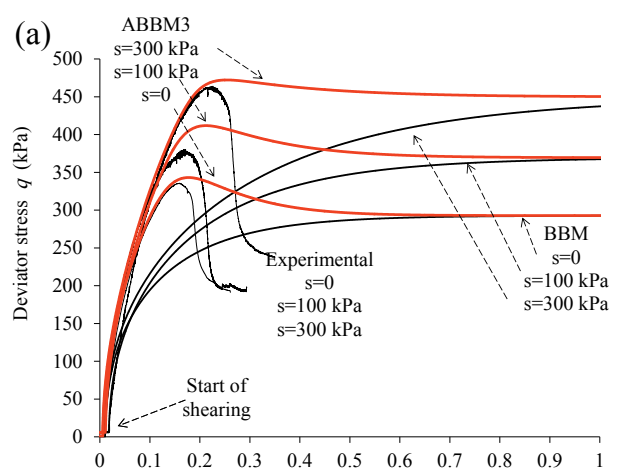

(a)

)

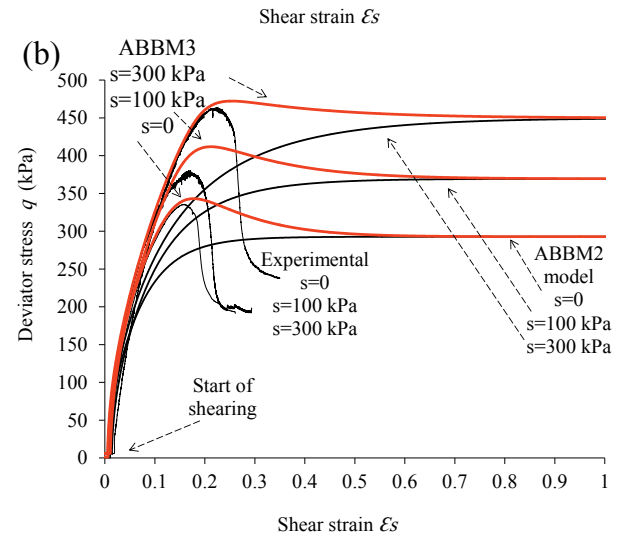

Figure 6. Experimental and predicted variation of $q$ against $\varepsilon_{s}$ : (a) ABBM3 vs BBM and (b) ABBM3 vs ABBM2. 

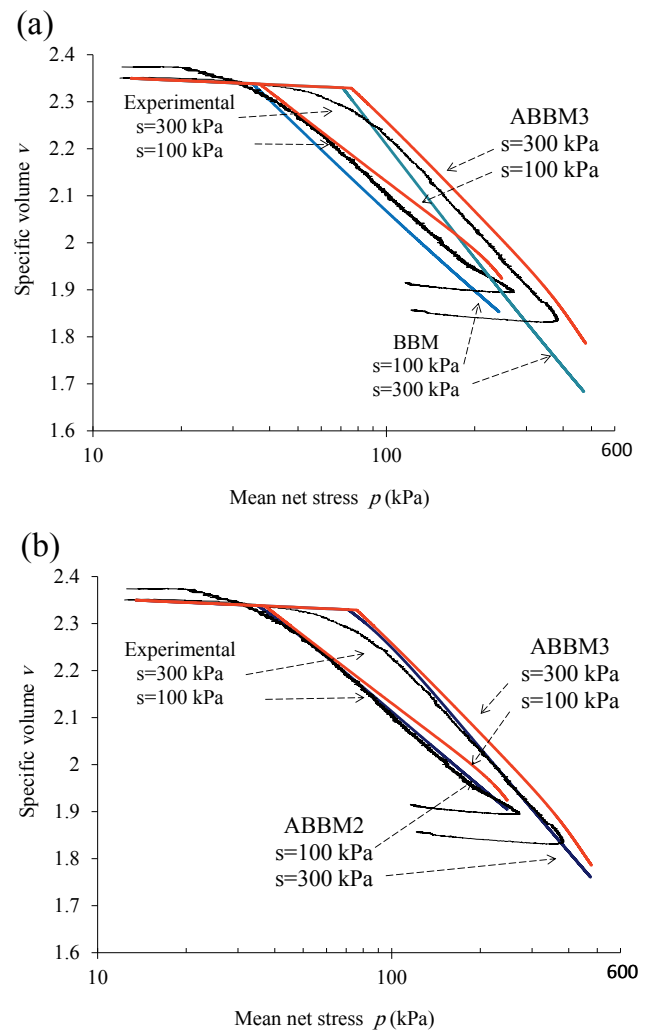

Figure 7. Experimental and predicted variation of $v$ against $\ln p$ : (a) ABBM3 vs BBM and (b) ABBM3 and ABBM2.
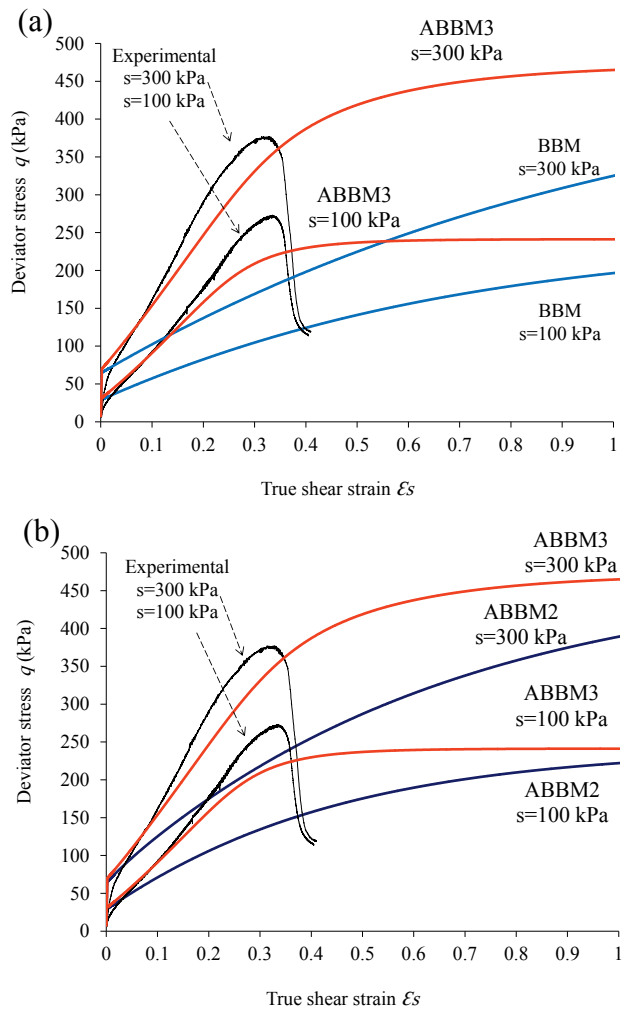

Figure 8. Experimental and predicted variation of $q$ against $\varepsilon_{s}$ : (a) ABBM3 vs BBM and (b) ABBM3 vs ABBM2.
In ABBM3, an improved form of elliptical yield surface with variable aspect ratio is proposed and an additional constitutive relationship is formulated between the aspect ratio and the distortion of the yield ellipse.

ABBM3 includes fifteen material parameters, ten of which are in common with BBM. The other five are necessary to define the evolution of anisotropy with plastic staining. This includes the change of both aspect ratio and distortion of the yield ellipse caused by plastic volumetric and shear deformations.

ABBM3 has been calibrated and validated against experimental data from Al-Sharrad [2]. Model simulations indicate that the incorporation of a variable aspect ratio in the mathematical formulation of the constant suction ellipse enhances the prediction of yield stresses and shear strains compared to BBM and ABBM2. Nevertheless, the number of model parameters becomes larger with increasing degree of model refinement, i.e. it increases from 10 in $\mathrm{BBM}$, to 12 in ABBM2 and to 15 in ABBM3.

Finally, the calibration of model parameters controlling evolution of anisotropy requires a large number of unconventional triaxial tests, which suggests that further simplification is necessary to facilitate the application of the model to engineering practice.

\section{References}

1. M. A. Al-Sharrd \& D. Gallipoli, Proceedings of the 6th International Conference on Unsaturated Soils, Sydney, Australia, 433-439, (2014).

2. M. A. Al-Sharrad, PhD thesis, University of Glasgow, UK, (2013).

3. J.H.A. Crooks \& J. Graham, Géotechnique, 26(2), 293-315, (1976).

4. J. Graham, M.L. Noonan \& K. V. Lew, Canadian Geotechnical Journal, 20(3), 502-516, (1983).

5. J. Graham, J.H.A. Crooks \& S.L.K. Lau, Géotechnique, 38(1), 125-134, (1988).

6. V. Sivakumar, I. G. Doran, J. Graham \& A. Johnson, Canadian Geotechnical Journal, 38(1), 125-137, (2001).

7. P.K. Banerjee \& N.B. Yousif, International Journal for Numerical and Analytical Methods in Geomechanics, 10, 521-541, (1986).

8. S. J. Wheeler, A. Naatanen, M. Karstunen \& M. Lojander, Canadian Geotechnical Journal, 40(2), 403-418, (2003)

9. V. Sivakumar, R. Sivakumar, E.J. Murray, P. Mackinnon \& J. Boyd, Géotechnique, 60(8), 581594, (2010).

10. V. Sivakumar, R. Sivakumar, J. Boyd \& P. Mackinnon, Géotechnique, 60(8), 595-609, (2010).

11. M. A. Al-Sharrad, S.J. Wheeler \& D. Gallipoli, Proceedings of the 2nd European Conference on Unsaturated Soils, Napoli, Italy, 2, 129-136, (2012).

12. K. Stropeit, S.J. Wheeler \& Y.J. Cui, Proceedings of 1st European Conference on Unsaturated Soils, Durham, UK, 625-631, (2008). 
13. F. D’Onza, D. Gallipoli \& S.J. Wheeler, Proceedings of the 5th International Conference on Unsaturated Soils, Barcelona, Spain, 2, 787-794, (2010).

14. G. Della Vecchia, C. Jommi \& E. Romero, International Journal for Numerical and Analytical Methods in Geomechanics, 37 (5), 503-535, (2012).
15. D. Gallipoli, F. D’Onza, \& S.J. Wheeler, Canadian Geotechnical Journal, 47(11), 1175-1186, (2010). 